\title{
Antidepressant Pharmacotherapy: Prescription Practices in Psychiatric Resident Care
}

\author{
Rachel Dew \\ Wake Forest University School of Medicine \\ W. Vaughn McCall \\ Wake Forest University School of Medicine
}

Follow this and additional works at: https://jdc.jefferson.edu/jeffjpsychiatry

Part of the Psychiatry Commons

Let us know how access to this document benefits you

\section{Recommended Citation}

Dew, Rachel and McCall, W. Vaughn (2005) "Antidepressant Pharmacotherapy: Prescription Practices in Psychiatric Resident Care," Jefferson Journal of Psychiatry. Vol. 19 : Iss. 1 , Article 4.

DOI: https://doi.org/10.29046/JJP.019.1.003

Available at: https://jdc.jefferson.edu/jeffjpsychiatry/vol19/iss1/4

This Article is brought to you for free and open access by the Jefferson Digital Commons. The Jefferson Digital Commons is a service of Thomas Jefferson University's Center for Teaching and Learning (CTL). The Commons is a showcase for Jefferson books and journals, peer-reviewed scholarly publications, unique historical collections from the University archives, and teaching tools. The Jefferson Digital Commons allows researchers and interested readers anywhere in the world to learn about and keep up to date with Jefferson scholarship. This article has been accepted for inclusion in Jefferson Journal of Psychiatry by an authorized administrator of the Jefferson Digital Commons. For more information, please contact: JeffersonDigitalCommons@jefferson.edu. 


\title{
Antidepressant Pharmacotherapy: Prescription Practices in Psychiatric Resident Care
}

\author{
Rachel Dew, M.D. and W. Vaughn McCall, M.D., M.S. \\ Wake Forest University School of Medicine
}

\begin{abstract}
Background: Research on treatment of depression has raised concerns regarding adequacy of medication trials and rationality of drug choice. Little data exists regarding pharmacotherapy by psychiatric residents. As practice habits begun in training will likely persist after graduation, examination of residents' antidepressant use may ultimately improve treatment by psychiatrists. Methods: Charts of new patients presenting to the Wake Forest University Psychiatry Resident Clinic were reviewed. Survey was made of medications prescribed to 112 patients diagnosed with major depression, dysthymia, depressive disorder NOS, adjustment disorder with depressed mood, or bipolar disorder with a documented depression during the studied period. Drug choice and maximum dose were noted. Results: Most-prescribed antidepressants included sertraline, trazodone, citalopram, mirtazapine, venlafaxine, and bupropion. The most used tricyclic antidepressant was amitriptyline $(n=7)$, with an average highest dose of $110.7 \mathrm{mg}$ per day. No MAOIs were prescribed. Augmentation treatment with lithium was prescribed twice and thyroid hormone once. No patients received ECT. Conclusions: Depressed patients in this resident clinic were treated primarily with SSRIs and other newer antidepressants. Little use was made of TCAs, MAOIs, ECT or traditional augmentation strategies. Further research should aim to determine whether more education in older antidepressant treatment modalities should be emphasized.
\end{abstract}

Keywords: Internship and Residency, Antidepressants

Key Words: internship, residency, antidepressants

\section{Introduction}

Research on the psychopharmacology of depressive disorders indicates that these illnesses are frequently undertreated by both psychiatrists and primary care providers $(2,3)$. Findings have questioned both typical dose and duration of antidepressant treatment, as well as the rationality of medication choice $(2,3)$. Depressive disorders carry a large burden of disability and suffering as well as contributing significantly to the risk of suicide; a recent study based on the National Comorbidity Study Replication found a 6.6\% 12month prevalence of major depression, with $59.3 \%$ of these cases demonstrating severe or very severe role impairment (1). As such, continued effort needs to be directed toward improving care for depressed patients.

In the push to improve the treatment of depression, it makes sense to consider how these patients are managed by psychiatric residents. Treatment principles learned during training will likely continue into postgraduate careers (4). Conversely, available treatment modalities not adequately learned in residency may never be subsumed into the practitioner's armamentarium. Research on psychiatric resident pharmacotherapy is scant. One study found that psychiatric residents are significantly influenced in medication decisions 
by contact with pharmaceutical representatives (5); other than this investigation, which addressed all classes of psychotropics, the authors know of no published study surveying the antidepressant prescribing practice of resident psychiatrists. Targeting deficiencies in resident education could have long-term benefits for the care of depressed patients.

Research on resident practice will also benefit those involved in medical education. In accordance with recent mandates by the Accreditation Council for Graduate Medical Education (ACGME), residency programs must document trainees' competency (6). For psychiatry residencies, this will include assessing psychopharmacology skills. Thus, initial surveys of current resident prescribing practices may prove useful in isolating problem areas and informing educational program design.

\section{Methods}

The study was approved by the Wake Forest University Baptist Medical Center (WFUBMC) Institutional Review Board. As the study involved retrospective chart review only, informed consent was not obtained. Charts of all new patients presenting to the WFUBMC Psychiatric Outpatient Department Triage Clinic January 1 through December 31, 2000 were reviewed; pharmacotherapy prescribed from the initial treatment up to 52 weeks of follow-up was assessed.

The Triage Clinic occurs weekly and serves as one entry point into outpatient psychiatric services at Wake Forest. From the initial assessment, nearly all patients enter the psychiatric resident outpatient clinic, which serves mainly indigent patients or those receiving federal aid. Patients live mainly in a small city or the surrounding rural areas.

The initial visit in the Triage Clinic is conducted by either a resident psychiatrist or family therapy intern, and observed by a single faculty psychiatrist and other trainees via one-way mirror. The clinic was supervised by the same attending for the entire period under study. From this visit, patients are generally referred to the resident medication clinic and followed by the same resident who conducted or observed the first interview. After the initial encounter, sessions are not observed by faculty; supervision occurs as needed at a weekly clinic case conference.

The clinic is staffed by house officers in the second, third, or, at times, fourth year of training. Several levels of training may be represented simultaneously, as the order of trainees' rotations depends in part on staffing needs and individual training goals. Patients in this study were treated by one resident in the second year of training, three who at the start of the studied period were in the second year and were followed into the third year, seven trainees in the third year, and one in the fourth year. Training years are summarized in Table 1.

\begin{tabular}{|c|c|}
\hline Treating Residents: & \\
\hline Women & 5 \\
\hline Men & 7 \\
\hline Treatment observed in: & \\
\hline 2nd year of training & 1 \\
\hline 2nd and 3rd year & 3 \\
\hline 3rd year & 7 \\
\hline 4th year & 1 \\
\hline
\end{tabular}

Table 1. Gender and post-graduate years of treating residents 
285 charts were reviewed; those that documented diagnoses by the treating residents of major depression, dysthymic disorder, depressive disorder NOS, adjustment disorder with depressed mood, or bipolar disorder with a depressed episode during the studied period were selected for further review. Diagnoses abstracted from the recorded chart were made by treating residents, using DSM-IV criteria via a one hour unstructured initial interview. Excluded subjects included 109 cases without a diagnosed depressive disorder, 18 primarily treated by attendings only, and eight referred elsewhere after the initial interview (mainly local public mental health centers). Also excluded were ten patients who received no pharmacological interventions (either because it was not felt to be indicated, the patient refused medication, or treatment was terminated prior to the initiation of pharmacotherapy). Eleven charts could not be located and 17 contained inadequate documentation by the treating resident of the diagnosis or prescribed medical regimen (e.g. no diagnosis documented, medication names or dosing unclear).

The 112 remaining charts were reviewed for up to 52 weeks of treatment following the initial visit. Review continued for as long as patients were retained (up to 52 weeks) regardless if depressive symptoms remitted; thus both initiation and maintenance-phase treatments were observed. Noted were drug choice and maximum dose, presence of comorbid psychiatric diagnoses, whether depression was classified as unipolar or bipolar, and presence of psychotic features. A Clinical Global Impression Severity score (CGI; a 1-7 scale with higher scores indicating more severe illness) was assigned to each case retrospectively by the reviewing author (RD); this score was assigned based on the most severe documented depressive symptomatology the patient displayed during the studied period.

Subsequent to chart review, the total occurrences for each observed medication was tallied, as well as the mean highest dose used. The mean highest dose was recorded to attempt to survey whether trainees titrated medications to a therapeutic dose; this gives some insight into whether medications were intended as primary treatment for depression or as adjuncts. Due to extreme variations in documentation of clinical decision-making, no attempt was made to code which psychotropics (many of which lay outside the antidepressant class) were used as treatments for depression and which for comorbid conditions.

\section{Results}

\section{A. Sample Characteristics}

The 112 studied patients had a mean age of 42.7 years +11.6 . Women made up $69.6 \%(n=78)$ of the sample, and $78.6 \%(n=88)$ were Caucasian. Twenty-three (20.5\%) were African American and one $(0.9 \%)$ was Asian. $92 \%$ of the sample had unipolar depression and $8 \%$ bipolar depression. Thirteen patients $(11.6 \%)$ had psychotic features. Further specifics of diagnosis, including recurrence status, were not coded. Comorbidities on Axis I occurred in 43.8\% ( $n=49)$ of cases and Axis II diagnoses in $12.5 \%(n=14)$. Substance abuse or dependence was diagnosed in 22 patients $(19.6 \%)$; otherwise, specific comorbid diagnoses were not recorded. The mean CGI Severity score was 3.8, corresponding to mild to moderate depressive symptomatology.

Patients studied were followed in the resident clinic for an average of $23.9+21.8$ weeks. $73.2 \%$ of patients arrived at the Triage visit already receiving medical treatment for depression, often prescribed by their primary care physician.

\section{B. Medications Prescribed}

For the total sample, 339 separate medication trials were undertaken, with a mean of $3.0+2.0$ medication trials per patient. Medications classified as antidepressants were prescribed in 219 trials. Table 2 lists all medications, how often they were used, and their mean highest daily dose. 
Most frequently prescribed antidepressants included sertraline ( $\mathrm{n}=38 ; 17.3 \%$ of all antidepressant trials), trazodone ( $n=29 ; 13.2 \%$ of antidepressant trials), citalopram ( $n=27 ; 12.3 \%$ of antidepressant trials), mirtazapine $(n=24 ; 11.0 \%$ of antidepressant trials), venlafaxine $(n=21 ; 9.6 \%$ of antidepressant trials), and bupropion ( $n=21 ; 9.6 \%$ of antidepressant trials). Paroxetine and nefazodone were prescribed to sixteen patients each (7.3\% of antidepressant trials each), and fluoxetine was utilized in eleven patients $(5.0 \%$ of antidepressant trials). Trazodone, although used often, was prescribed mainly at lower doses (mean highest daily dose $=103.4 \mathrm{mg}$ ) as needed for insomnia.

Tricyclic antidepressants were used infrequently. Most often used was amitriptyline, prescribed to seven patients (3.2\% of antidepressant trials), with a mean highest daily dose of $110.7 \mathrm{mg}$. No monoamine oxidase inhibitors were prescribed.

Augmentation of an antidepressant with lithium occurred in two patients. Thyroid hormone was prescribed to one patient as an augmentor. Most common adjunctive agents used were clonazepam $(n=27 ; 8.0 \%$ of all psychotropic trials), zolpidem ( $n=17 ; 5.0 \%$ of all psychotropics), and alprazolam ( $n=16 ; 4.7 \%$ of all psychotropics). No patients in the sample received electroconvulsive therapy during the studied period.

\begin{tabular}{|c|c|c|}
\hline Medications & Occurrences & Mean highest dose (mg) \\
\hline venlafaxine (extended release) & 21 & 87.5 \\
\hline citalopram & 27 & 24.8 \\
\hline sertraline & 38 & 102.6 \\
\hline amitriptyline & 7 & 110.7 \\
\hline quetiapine & 6 & 183.3 \\
\hline clonazepam & 27 & 1.9 \\
\hline zolpidem & 17 & 10 \\
\hline doxepin & 5 & 50 \\
\hline trazodone & 29 & 103.4 \\
\hline bupropion (sustained release) & 21 & 250 \\
\hline lithium & 2 & 750 \\
\hline nefazodone & 16 & 287.5 \\
\hline paroxetine & 16 & 27.5 \\
\hline mirtazapine & 24 & 29.4 \\
\hline valproate & 6 & 791.7 \\
\hline buspirone & 6 & 32.5 \\
\hline risperidone & 8 & 1.9 \\
\hline lorazepam & 6 & 2.1 \\
\hline alprazolam & 16 & 2.6 \\
\hline imipramine & 3 & 81.7 \\
\hline gabapentin & 5 & 780 \\
\hline clonidine & 2 & 0.1 \\
\hline haloperidol & 1 & 2 \\
\hline ziprasidone & 2 & 100 \\
\hline fluoxetine & 11 & 39 \\
\hline olanzapine & 8 & 4.7 \\
\hline hydroxyzine & 2 & 100 \\
\hline diphenhydramine & 1 & 50 \\
\hline diazepam & 1 & 10 \\
\hline nortriptyline & 1 & 25 \\
\hline methylphenidate & 2 & 22.5 \\
\hline amphetamine/dextroamphetamine & 1 & 5 \\
\hline cytomel & 1 & 0.015 \\
\hline
\end{tabular}




\section{Discussion}

Two of the three most often prescribed antidepressants in this resident clinic sample were SSRIs; this conforms with a recent survey which found $93 \%$ of psychiatrists felt SSRIs were first-choice treatment (7). Also frequently utilized were the other newer antidepressants bupropion, mirtazapine, and venlafaxine.

Trazodone, although prescribed often, seemed to be used predominantly with other agents and intended as a hypnotic. This finding is consonant with a study of psychiatrist and primary care antidepressant treatment which found that of 241 trials of trazodone, only 3 were considered adequate (defined as daily dose $>300$ $\mathrm{mg}$ ) (3). Most likely, the bulk of these trials, deemed as "inadequate," were actually intended to be adjunct trials for depression-related insomnia.

A noteworthy finding is the lack of employment of older modalities with proven efficacy. TCAs were used rarely and in doses that would usually be considered inadequate (3). MAOIs were not prescribed to any of the 112 patients. Lithium and thyroid hormone augmentation were strategies used very seldom, and no use was made of electroconvulsive therapy.

These trends, too, may be consistent with general practice. Weilburg et al.'s study found only $27 \%$ of TCA trials achieved adequacy in terms of dosage level and duration of treatment, compared with 51\% adequacy using the SSRIs. A 1999 study on the use of MAOIs found that only $2 \%$ of psychiatrists described prescribing them frequently (8). Another survey found that psychiatrists felt newer agents to be more efficacious than TCAs and MAOIs despite a lack of scientific evidence to support this (7).

\section{A. Study Limitations}

Before considering the implications of these results, note should be made of this descriptive, retrospective study's several limitations and possible sources of bias. Firstly, we must assess whether our results adequately sampled the treatment usually received in this resident clinic. Multiple factors may have influenced the results. Seventeen of the original 285 new patient charts $(6 \%)$ contained little or no documentation; thus, it could not be discerned whether these seventeen patients suffered depressive disorders, and, if so, may have been treated in a way that differed significantly from the 112 reviewed charts. (However, one might assume that a resident using a treatment modality very different from their usual practice would document this clearly.) The same caveat applies to the eleven charts which had been lost. It is also possible that those twenty-six cases referred to staff psychiatrists or to other treatment agencies (mainly local mental health centers) presented a significantly different clinical picture-perhaps featuring severe treatment resistance - and would have been treated in an appreciably different manner than the retained patients.

Another possible biasing factor on medications used in the study is time retained in the clinic. This varied widely $(23.9+21.8$ weeks $)$ in the reviewed cases. The review was truncated at 52 weeks of follow-up from the initial presentation. In addition, a few $(<10)$ patients stopped coming before a medication trial was initiated. If patients were retained longer, or if chart review had been extended beyond 52 weeks, patients may have been found to have received different treatments - quite possibly treatments not considered firstline (such as older antidepressants).

It must also be remembered that no attempt was made to correlate medication choice with illness severity or treatment resistance. The average severity of illness in the sample, represented by the CGI Severity scores assigned retrospectively, was mild to moderate. Possibly this sample did not contain cases sufficiently treatment resistant to merit trials of older modalities. However, it is also true that a high proportion of patients had a medication trial prior to referral, indicating that some degree of treatment resistance was present. 
Finally, the study is limited in that it does not assess non-pharmacologic treatment of depression i.e. psychotherapy. Most residents must meet specific requirements by carrying several psychotherapy cases; we may assume residents use psychotherapy as the primary treatment for depression in some proportion of cases.

Another possible concern is that these medication choices were not made by the residents independently, and thus don't represent their true treatment preferences. For example, a high proportion (73.2\%) of patients arrived on treatment initiated elsewhere, often by their primary care providers. Whether the treating resident continued the medication already initiated elsewhere was not recorded in chart review; quite possibly the medication prescribed by the outside care provider was continued in a high percentage of cases. However, the results should still reflect to some degree whether the treating resident felt the outside provider's medication choice was appropriate.

Another possibility is that medications used primarily reflect the preferences of the residents' faculty mentors rather than their own. The first interview of all patients was observed by a single faculty psychiatrist who supervised the triage clinic for the entire study period. It is probable that treatment preferences of this attending psychiatrist exerted significant influence on which medications residents prescribed. Their choices were also most likely influenced by prescription practices of other faculty supervisors they worked with in the program. This should not, however, necessarily be considered a weakness, as the study's aim was to describe what prescription practices trainees learn in a residency program-where working with supervisors is a primary educational vehicle.

\section{B. Implications}

Despite the limitations of the study, if we assume our results offer a fair depiction of psychiatric house officer treatment preferences, how do we explain trainee medication choice? One influence may be the pharmaceutical industry, which promotes the advantages of newer, brand-name agents at times by emphasizing relative disadvantages of older agents such as the tricyclic and heterocyclic agents and MAOIs. This has been found to be a clinically significant factor (5). Another consideration is the availability in the clinic of samples of brand-name agents; the option of dispensing free samples may well influence treatment decisions, especially when servicing indigent patients.

Alternatively, it may be that older modalities such as TCAs and MAOIs were consciously discarded in residents treatment practice due to the expectation of poor tolerability. Or, possibly, trainees may have considered use of older agents in resistant cases but shied away from them due to discomfort and unfamiliarity with their use.

Should this last possibility prove the case, and should this resident clinic sample prove typical of general practice by psychiatric house officers, these findings beg the question: should residents be learning to use older antidepressant treatments? They have not disappeared from the curriculum, but they are not being prescribed by residents. The paradigm underlying medical education states that one learns in practice; if residents do not practice and gain facility using older modalities, are they likely to later feel comfortable using them? Alternatively, one might argue that some of these treatments, due to the ever increasing availability of efficacious and better tolerated medications, are becoming obsolete-and therefore no longer need to be learned well.

Despite indications that TCAs and MAOIs are being used less, the Texas Medication Algorithm Project consensus for major depressive disorder without psychotic features places them in "Stage 2" and "Stage 3 " respectively, as alternative medications after initial treatment failure (9). If this algorithm represents a psychiatric standard of care, it would seem that the specialty should expect residents to become facile with these agents. 
Research has demonstrated promise with the use of treatment algorithms to improve treatment of depressive disorders, despite some reluctance by physicians to use the preset algorithm (10). Perhaps specific training in treatment algorithms in residency, as less experienced clinicians may be more receptive to their use, would ultimately improve treatment of depressive disorders. This approach may also help ensure that all standard pharmacotherapeutic strategies are adequately learned. This could help residency programs ensure trainees reach core competency in psychopharmacology, which, as mentioned in the introduction, will need to be documented for compliance with ACGME regulations.

In sum, the residents in this clinic treated depressed patients predominantly with SSRIs and other newer antidepressants. Little use was made of TCAs, MAOIs, ECT or augmentation with lithium or thyroid hormone. Most frequently prescribed adjunctive treatments included benzodiazepines and hypnotics. Future research should evaluate trends in resident practice in order to determine what, if any, changes in the educational process should occur with regard to older antidepressant strategies.

\section{REFERENCES}

1. Kessler RC, Berglund P, Demler O, et al. The epidemiology of major depressive disorder: results from the National Comorbidity Survey Replication (NCS-R). JAMA 2003 ; 289 (23): 3095-105.

2. Insel TR, Charney DS. Research on major depression: strategies and priorities. JAMA 2003; 289 (23): 3167-3168.

3. Weilburg JB, OLeary KM, Meigs JB, et al. Evaluation of the adequacy of outpatient antidepressant treatment. Psychiatric Services 2003; 54 (9): 1233-1239.

4. Awad AG, Darby PL, Garfinkel PE: Psychopharmacology training in psychiatric residency programs: the Canadian scene. Canadian J Psychiatry 1991; 36: 21-25.

5. Schwartz TL, Kuhles DJ 2nd, Wade M, Masand PS. Newly admitted psychiatric patient prescriptions and pharmaceutical sales visits. Ann Clin Psychiatry 2001; 13 (3): 159-62.

6. ACGME Outcome Project: Enhancing residency education through outcomes assessment. Retrieved February 16, 2003 from http://www.acgme.org/outcome/.

7. Petersen T, Dording C, Neault NB, et al. A survey of prescribing practices in the treatment of depression. Prog Neuropsychopharmacol Biol Psychiatry 2002; 26 (1):177-87.

8. Balon R, Mufti R, Arfken CL. A survey of prescribing practices for monoamine oxidase inhibitors. Psychiatric Services 1999; 50 (7): 945-957.

9. Trivedi MH. Using treatment algorithms to bring patients to remission. J Clin Psychiatry 2003; 64 (suppl 2): 8-13.

10. Adli M, Berghofer A, Linden M, et al. Effectiveness and feasibility of a standardized stepwise drug treatment regimen algorithm for inpatients with depressive disorders: results of a 2-year observational algorithm study. J Clin Psychiatry 2002; 63 (9): 782-90. 